\title{
IDENTIDAD ÉTNICA COMO UN DERECHO CULTURAL
}

\author{
Isi Verónica Lara Andrade ${ }^{1}$ \\ José Antonio Morales Notario ${ }^{2}$ \\ Josefina De la Cruz Izquierdo ${ }^{3}$
}

Resumen: La identidad es indisociable de la cultura y sostiene que los humanos pertenecemos de ciertos repertorios culturales; la identidad es el lado subjetivo de la cultura y se constituye en virtud de un juego dialéctico entre la autoafirmación y la diferencia (Pérez Castro, (2012). Por lo cual, tiene una dimensión civilizatoria, puesto que representa el hacer de las identidades. Así, los procesos de construcción política y civilizatoria de las sociedades multiétnicas se producen como resultado de la articulación de identidades y de culturas diferenciadas. Puede basarse en la cultura, pero no depende de un patrimonio exclusivo; incluso las tradiciones culturales pueden inventarse, reconstruirse y apropiarse, sin que deje de constituir bases para la formulación identitarias (Bartolomé, (2006). Por ejemplo, la Virgen de Guadalupe, independientemente de que forma parte de la religión católica para los mexicanos, es un símbolo de identidad, sean católicos o no. La cultura como manifestación social está sujeta al cambio, y éste, dependiendo de las circunstancias, puede ser a corto plazo (o relativamente rápido) o a muy largo plazo. Por eso, la cultura puede ser vista de dos formas: como una herencia, una tradición y una persistencia, y también se puede ver como desviación, innovación y metamorfosis permanente (Gámez Espinoza, (2015). La cultura hace la diferencia entre las sociedades, generando sentido de pertenencia y legitimando a una colectividad. Al igual que la cultura se crea también a partir de nuestras relaciones con los demás, con las cosas y las situaciones, pues nos construimos una idea basados en la realidad. De ahí que la identidad está conformada por factores imprescindibles como el de territorialidad, consanguinidad, lengua, religión y política, los que

\footnotetext{
${ }^{1}$ Dra. en Derecho. Profesora Investigadora de la Universidad Juárez Autónoma de Tabasco

${ }^{2}$ Dr. en Derecho. Profesor Investigadora de la Universidad Juárez Autónoma de Tabasco

${ }^{3}$ Dr. en Educación. Profesora Investigadora de la Universidad Juárez Autónoma de Tabasco
} 


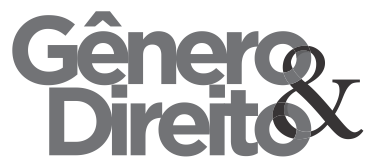

Periódico do Núcleo de Estudos e Pesquisas sobre Gênero e Direito Centro de Ciências Jurídicas - Universidade Federal da Paraíba V. 6 - No 03 - Ano 2017 (Spanish Edition) ISSN | 2179-7137 | http://periodicos.ufpb.br/ojs2/index.php/ged/index permiten determinar la pertenencia a una comunidad indígena, y que a pesar del fenómeno migratorio que les precede, sigue determinando la pertenencia e identidad de los indígenas.

Palabras Claves: Identidad Cultural, Pueblos indígenas, etnias, derecho cultural

Abstract: Identity is inseparable from culture and holds that humans belong to certain cultural repertoires; Identity is the subjective side of culture and is constituted by virtue of a dialectical game between self-assertion and difference (Pérez Castro, 2012), and therefore has a civilizing dimension, since it represents the making of identities. Thus, the processes of political and civilizing construction of the multiethnic societies are produced as a result of the articulation of different identities and cultures. It can be based on culture, but it does not depend on an exclusive patrimony, even cultural traditions can be invented, reconstructed and adjudged. For example, the Virgin of Guadalupe, regardless of whether she is part of the Catholic religion for Mexicans, is a symbol of identity, whether Catholic or non-Catholic (Bartolomé, 2006).
Culture, as a social manifestation, is subject to change, and this, depending on the circumstances, may be short-term (or relatively rapid) or very long-term. This is why culture can be seen in two ways: as a heritage, a tradition and persistence, and it can also be seen as a deviation, innovation and permanent metamorphosis (Gámez Espinoza, (2015).) Culture makes the difference between societies, generating a sense of belonging and legitimating a community. Culture is also created from our relationships with others, with things and situations, as we build an idea based on reality. Thus, identity is made up of essential factors such as territoriality, consanguinity, language, religion and politics, which determine the membership of an indigenous community, and despite the migratory phenomenon that precedes them, continues to determine the identity of the indigenous people.

Keywords: Cultural identity, indigenous peoples, ethnicity, cultural right.

\section{INTRODUCCIÓN}

¿Que entendemos por identidad? Los rasgos particulares que van a 


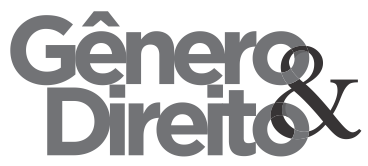

diferenciar un grupo social de otro, pero de los grupos donde sus características son comunes estos rasgos le dan pertenencia a ese grupo en particular. El término identidad tiene varias concepciones en el sentido socio-cultural, es un conjunto de valores, símbolo, lengua, creencias y costumbres de una cultura que se diferencia de otras. Estos se presentan como elementos cohesionadores de un grupo social al fundamentar su sentimiento de pertenencia (Pérez Monroy, (2012).

Elementos en común como: las acciones, ideas y sentimientos que definen a individuos o comunidades como parte de un determinado tiempo, espacio, grupo, de una forma de pensar y de sentir. Pero en la convivencia humana se presentan también fenómenos opuestos, lo que diferencia a individuos o a grupos sociales. Aquellos que no comparten una identidad se le denomina otredades, o alteridad, según Sartori, nosotros somos quienes somos, y como somos, en función de quienes o como no somos. Estas otredades o alteridad es el supuesto de la identidad nacional y la indígena (Pérez Monroy, (2012).

\section{IDENTIFICACIÓN DE LOS PUEBLOS INDÍGENAS}

Carlos Durand señala que la identidad de las poblaciones indígenas en México no proviene tan sólo de la importancia que tiene para el grupo asimilación-identificación y un asumir de su idioma, tradiciones, cosmogonía y otros aspectos culturales; como el territorio que es imprescindible para la reproducción cultural, y en muchas poblaciones se conserva el sistema comunal como elemento de identidad y cohesión social (Durand Alcántara, (2006).

La identidad se explica desde las cincos perspectivas que cita la autora Mariflor Aguilar: la primera es la transindividual, es decir, es la trama compleja de relaciones que se establecen entre el individuo y los otros, y entre el individuo y las prácticas sociales; el sentido de pertenencia es el hecho de que alguien es parte de un grupo, pertenece a él, como también refiere a un sentimiento, a la vivencia que tienen los individuos de sentirse o no parte de algo. La Segunda tesis, la identificación, sostiene que son tres los registros fundamentales que sustentan las relaciones humanas con el 
mundo, entre ellas la imaginación que identifica a los individuos con la imagen de sus semejantes. La Tercera tesis señala que la identidad no es algo dado de una vez y para siempre, sino que es móvil, son identificaciones que están siempre en proceso de reacomodo y de reestructuración jerarquizada. La Cuarta, las desidentificaciones, son los procesos cuando una identificación debe ser removida con el fin de que otra vaya tomando su lugar, las experiencias migratorias serian ejemplo de estos casos. Otra forma es la negación de una identidad y; la Quinta y última tesis es la de identificación múltiple, es decir que no hay una identificación única (Aguilar Rivero, (2012).

La identidad es un elemento que define la relación de la etnia con su territorio en la medida que se establece un sentido (sentimiento) de pertenencia en ese espacio geográfico y en su entorno socio cultural, este sentir se ha transmitido de generación en generación y se encauza y concibe como la defensa y respeto de estas posesiones legadas por los ancestros (Durand Alcántara, (2005). El etnólogo Lévi-Strauss sostiene que "se requiere la identidad porque sin ella se renuncia a todo tipo de compresión e incluso de diálogo con el otro, puesto que hasta el diálogo con el otro, supone un mínimo de identidad".

Parte de la identidad personal descansa sobre metas y actividades que constituyen un plan de vida. Las etnias también tienen un plan de vida pero éste se desarrolla en común y se conoce como identidad colectiva. Dentro de la Identidad indígena el sujeto implicado se desarrolla en un contexto cultural que le permite sentirse involucrado con sus manifestaciones más representativas como pueden ser el idioma, las tradiciones, los usos y costumbres; haciendo de todo ello su perfil representativo y de manifestación ante el mundo externo; por lo que es muy probable que conlleven a formar un elevado sentido de solidaridad con su comunidad (Pérez de los Reyes, (2013).

Las actitudes relacionadas con los fenómenos de la cultural material están mediatizadas por la cosmovisión y la espiritualidad indígena de mentalidad tradicional. Se da una relación íntima, profunda y respetuosa entre el hombre y las potencias del mundo sobrenatural y de la Naturaleza Silvestre. Pues se cree que estos mundos están habitados y animados por espíritus cuya ponencia del mundo 
sobrenatural y de la naturaleza silvestre, residen en ciertas plantas, árboles y animales, como también en ciertos fenómenos geológicos, atmosféricos o astrales. En consecuencias, el indígena tradicional interactúa con espíritus vinculados con fenómenos naturales y culturales. Se cree que tanto la tierra como el agua, tanto el cerro como el bosque y el árbol, los animales y pájaros silvestre, la semilla y la siembra, la piedra, el camino tropero, el ganado, el tejido a telar -todos ellos- poseen espíritu, vida propia y poder. Las diferencias entre los grupos socioculturales son marcadas por la pertenencia a cierta etnia y distingue a los miembros de una comunidad indígena (Grebe Vicuña, (2010).

\section{LA CULTURA COMO BASE DE LA IDENTIDAD ÉTNICA}

La palabra cultura, sostiene el sociólogo Bauman, ingresó en el vocabulario moderno como una declaración de intenciones, como el nombre de una misión que aún era preciso emprender. En sus comienzos a la idea de cultura se le designaron tres características: "optimismo, es decir, la creencia de que el potencial para el cambio en la naturaleza humana es ilimitado; universalismo, es decir, el supuesto según el cual la idea de naturaleza humana y el cumplimiento potencial de sus exigencias son iguales para todas las naciones, todos los lugares y todos los tiempos; y por último, eurocentrismo, es decir, la convicción de que ese ideal se había descubierto en Europa".

A partir de esta concepción, los estados querían occidentalizar la cultura, reemplazando la cultura de las comunidades que tienen diversas tradiciones, costumbres y lenguas, para crear un estado homogéneo, sin saber que la verdadera igualdad radica precisamente en el respeto de esas diferencias culturales, por lo cual merecen existir por la simple razón de sus diferencias (Bauman, (2013).

La UNESCO estableció que la diversidad cultural "se refiere a la multiplicidad de formas en que se expresan las culturas de los grupos y sociedades". De acuerdo a la Declaración Universal sobre la Diversidad Cultural, "la cultura debe ser considerada como el conjunto de los rasgos distintivos espirituales y materiales, intelectuales y afectivos que caracterizan a una sociedad 
o a un grupo social", abarca "los modos de vida, las maneras de vivir juntos, los sistemas de valores, las tradiciones y las creencias" (Díaz-Polanco, (2009).

Por otra parte, la autora Milka Castro cita al filósofo Polaco Bauman, quien señala que: "la cultura se entiende como algo que uno posee o como un proceso que uno moldea. Se tiene la creencia de que las características se han configurado a través de largos procesos históricos y en la actualidad se piensa que influyen e incluso dan forma a los actos y a la manera de pensar de todos los llamados miembros. Bajo este prisma, la cultura, ya sea nacional, étnica o religiosa, es algo que uno posee y de la que uno es miembro, y no algo que uno crea y moldea a través de la constante actividad renovadora" (Castro Lucic, (2004).

\section{FACTORES QUE DETERMINAN LA IDENTIDAD}

Cuando se habla de factores se está utilizando el término en sentido de las circunstancias que determinan en este caso a la identidad. En el momento que se determinan estas circunstancias 0 elementos se entra al estudio de ellos y se van manifestando en una determinada comunidad. El conjunto de estos factores dan el sentido de pertenencia al individuo, formando la personalidad a través de sus creencias, ideologías e ideas como el territorio, el parentesco, la lengua, la política y la religión (creencias y ritos), creando vínculos que llevan a definir la identidad.

En su sentido etimológico, el territorio, que es para los grupos étnicos uno de los rasgos más elementales y, según la Real Academia de la Lengua Española, “deriva del latín territorium y se utiliza para denominar a una porción de la superficie terrestre perteneciente a una nación, región, provincia, etc. De acuerdo al art. 13 del Convenio sobre Pueblos Indígenas y Tribales (1989), el concepto de territorio se refiere a lo que cubre la totalidad del hábitat de las regiones que los pueblos interesados ocupan o utilizan de alguna otra manera.

En la Declaración de las Naciones Unidas sobre los Derechos de los Pueblos Indígenas (2007), se especifica que los pueblos indígenas tienen el derecho de mantener y fortalecer su propia relación espiritual con tierras, territorios, aguas, mares costeros y otros recursos que 
tradicionalmente han poseído u ocupado y utilizado y a asumir las responsabilidades que les corresponden con las generaciones venideras. Dichos Pueblos Indígenas poseen de manera colectiva la propiedad en donde viven, y esto permite medir como un indicador efectivo el desarrollo humano indígena, determinando los ingresos económicos de los Pueblos Indígenas (Batzin, 2005).

En cuanto se refiere al parentesco, esta palabra viene de pariente, y éste, a su vez, del latín parens-entis y se conoce como el vínculo existente entre las personas que descienden unas de otras o de un progenitor común, corresponde a la realidad biológica y en sí, al hecho de la procreación. El derecho toma en cuenta estas fuentes primarias de la relación humana y crea otras más, independientemente de los datos biológicos, para configurar su propio concepto que es la relación jurídica que se establece entre los sujetos en razón de la consanguinidad, de la afinidad o adopción (Instituto de Investigaciones Jurídicas, (s. f.). Es decir el vínculo por consanguinidad, afinidad, adopción, matrimonio u otra relación estable de afectividad análoga a ésta. Se establece como una característica central de los pueblos indígenas, pues el parentesco de sangre y los antepasados comunes son los vínculos que sostienen la cohesión y la pertenencia a una gran familia que evidencian su autenticidad (Bello, 2004). La lengua es un factor distintivo en un grupo indígena y según la Real Academia Española (RAE) es el modo de expresión de una comunidad, porque las personas que hablan una misma lengua conforman una comunidad lingüística, lo que significa que dicho colectivo o grupo maneja el mismo vehículo de comunicación y expresión.

En el Art. II sección segunda, apartado 2 del Proyecto de Declaración Americana sobre los Derechos de los Pueblos Indígenas dice: "Los pueblos indígenas tienen los derechos colectivos que son indispensables para el pleno goce de los derechos humanos individuales de sus miembros. En ese sentido, los Estados reconocen el derecho de los pueblos indígenas, entre otros, a su actuar colectivo, a sus propias culturas, de profesar y practicar sus creencias espirituales y de usar sus lenguas", y en el convenio 169 de la Organización Internacional del Trabajo, también se les da reconocimiento para mantener sus 
identidades, lenguas y religiones dentro del marco de los Estados en que viven (Bello, 2004). La política se identifica y define en un pueblo como el Arte o traza con que se conduce un asunto o se emplean los medios para alcanzar un fin determinado o actividad del ciudadano cuando interviene en los asuntos públicos con su opinión, con su voto, o de cualquier otro modo. También Bernard Crick en su estudio "En defensa de la política" (1962), según Catherine Martínez (s. f.) define que la política es la actividad que concilia los intereses de distintos individuos a través de la división proporcional del poder.

En el artículo 5 de la Convención Internacional sobre la eliminación de todas las formas de Discriminación Racial ( 1965), se prohíbe y elimina la discriminación racial en todas sus formas, garantizando el derecho de toda persona a la igualdad ante la ley, por lo que se protegen los derechos políticos, el de tomar parte en elecciones, elegir y ser elegido, por medio del sufragio universal y de participar en el gobierno y dirección de los asuntos públicos en cualquier nivel y en condiciones de igualdad a las funciones públicas. Por lo que los Estados se han comprometido en reconocer, respetar y promover estas formas de organización propias de las comunidades indígenas, que reconozcan el papel que las autoridades de las comunidades, constituidas acorde a sus normas consuetudinarias, en el manejo de sus asuntos y la toma de decisiones de acuerdo a sus propias prioridades y su proceso de desarrollo, educación, salud, cultura e infraestructura (Naciones Unidas (1995). Y por último la religión, la cual según la Real Academia Española de la Lengua, (del lat. Religió,-onis) la define como el conjunto de creencias o dogmas acerca de la divinidad, de sentimiento de veneración y temor hacia ella, de normas morales para la conducta individual y social y de prácticas ritualistas, como la oración y el sacrificio para darle culto (Real Academia Española, (s. f.).

Dentro del Acuerdo sobre identidad y derechos de los pueblos indígenas se determina que la espiritualidad es un componente esencial de su cosmovisión y de la transmisión de sus valores y de los demás pueblos indígenas, por lo que los Estados se comprometen a hacer respetar el ejercicio de esta espiritualidad en todas sus manifestaciones y su derecho a practicarla en público y privado por medio de la 


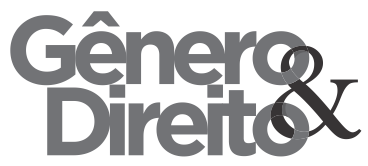

enseñanza, culto y observancia, respetando a sus guías espirituales indígenas, sus ceremonias y sus lugares sagrados (Naciones Unidas, (1995).

\section{TERRITORIALIDAD,}

\section{PARENTESCO, LENGUA, Y POLÍTICA}

Para desarrollar y entender mejor los factores que determinan la identidad de los pueblos indígenas ahondaremos de manera específica en cada uno de ellos, iniciando con el de la importancia de la tierra; es el punto cohesionador de la misma, la que con su entorno-naturaleza permite reproducirla. Es pues, el escenario en donde se desenvuelven las formas de producción y de cultura propio; no solo se concibe en el sentido económico, esto es, como un medio de producción, sino como el asentamiento y base de su existencia espiritual (Durand Alcántara, (2005).

Los incas, la veneran y la identifican como la Pachamama, la madre tierra. La territorialidad es el punto más importante, en efecto, el derecho a la posesión de la tierra está garantizado en varios artículos que se encuentran en los diversos instrumentos internacionales, por
Céco do Núcleo de Estudos e Pesquisas sobre Gênero e Direito Centro de Ciências Jurídicas - Universidade Federal da Paraíba V. 6 - No 03 - Ano 2017 (Spanish Edition)

ejemplo la Corte Interamericana señala que "para las comunidades indígenas la relación con la tierra no es meramente una cuestión de posesión y producción sino un elemento material y espiritual del que deben gozar plenamente", incluso argumenta, que es factor imprescindible para conservar su legado cultural y poder transmitirlo de generaciones en generación (Naciones Unidas, (1995).

Es indudable que para estos pueblos el territorio representa un referente fundamental dentro del cual inscribir la identidad colectiva en la medida en que la ideología social se construye y en relación con el medio ambiente determinado (Bartolomé, (2006). Esto se puede apreciar cuando se dan los primeros asentamientos, el ecosistema tuvo mucho que ver en las formas en que estos pueblos se organizaron y desarrollaron.

En cuanto al territorio, para el Derecho Internacional de los Derechos Humanos, se debe garantizar que, en cualquier momento que se vaya a tomar decisiones sobre las tierras de los pueblos originarios, éstos deben estar informados y tengan posibilidades de participación dentro de las decisiones. Entender el 


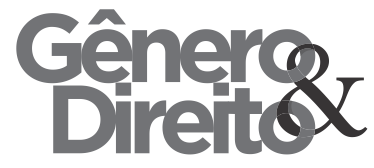

territorio como consecuencia de determinadas relaciones sociales, políticas y económicas nos permite reflexionar sobre el rol y la significación que el parentesco tiene en su construcción (Andrea Maidana, (2009).

Los sistemas parentales ocupan un papel importante en la formación identitaria colectiva. En casi todos los grupos, dichas relaciones son determinantes para la filiación étnica. Sobre todo en aquellas sociedades clánicas, por ejemplo los triquis se definirían como tal en función de su pertenencia a algunos de los clanes territoriales de su sociedad, de igual manera para los zoques, es más importante la filiación que la lengua (Bartolomé, (2006). Posiblemente se vean los lazos de parentesco como simples vínculos afectivos, como lo señala Carolina Andrea, sin embargo son indispensables en materia de afiliación e identidad, pues crean vínculos entre el presente y el pasado, y permite vislumbrar la relación del futuro, haciendo que los nacidos en la ciudad se reconozcan e identifiquen en una trayectoria, un origen y una historia común, consolidando el sentido de pertenencia a un colectivo ya sea por
Céco do Núcleo de Estudos e Pesquisas sobre Gênero e Direito Centro de Ciências Jurídicas - Universidade Federal da Paraíba V. 6 - No 03 - Ano 2017 (Spanish Edition)

consanguinidad o alianza (Andrea

Maidana, (2009).

Por ello, el indígena se reconoce entre sí, aunque hayan olvidado la lengua, porque son sabedores de sus antepasados y de su árbol genealógico, filiación que le otorga identidad étnica. Sin embargo, la identidad de un grupo socio-cultural también se encuentra en el idioma, es decir su lengua que es medio de identificar como indios a los miembros de un grupo o conglomerado indígena. Cuando se habla de los chontales, tarahumaras, nahuas, mixes, etc., con esas denominaciones, es porque tales grupos hablan las lenguas de esos nombres (Pozas y De Pozas, (2006). México es el segundo país con mayor diversidad de lenguas, existen 11 familias lingüísticas, las cuales se subdividen en 68 agrupaciones lingüísticas, que a su vez incluyen un total de 364 variantes lingüísticas actualmente registradas. $\mathrm{Si}$ consideramos a la población hablante de una lengua indígena, el grupo mayoritario está integrado por quienes hablan náhuatl, los cuales representaban en 2010 el $23 \%$ de la población indígena del país. En orden descendente, le siguen los hablantes de maya $(11.5 \%)$, tzeltal (7\%), mixteco $(6.9 \%)$ tzotzil $(6.5 \%)$, zapoteco $(6.4 \%)$, 
otomí (4.2\%), mazateco (3.5\%), totonaca (3.3\%), ch'ol (3.1\%) huasteco $(2.5 \%)$ y chinanteco $(2.1 \%)$. En conjunto, estas lenguas dan cuenta del $80 \%$ de la población hablante de alguna variedad lingüística indígena (Bertely Busquets, et, al., (2013).

Sin embargo con el proceso de aculturación con las políticas del liberalismo al tratar de unificar a todos los habitantes de México, en las escuelas se exigía que aprendieran el castellano y debido a ello muchos habitantes de los pueblos indígenas han ido perdiendo su lengua materna, lo que algunos autores consideran como la pérdida de su identidad. En la obra Derechos de Indios en México, Carlos Durand sustenta que se debe de reconocer de manera real y formal -jurídicamente- a sus lenguas, porque, en ello radica la permanencia de sus expresiones sociales, culturales y de índole diversa (Durand Alcántara, (2005). Por ello, el Estado creo políticas obligatorias en torno a la conservación y preservación de la lengua en las escuelas bilingües, publicando, así, la Ley General de Derechos Lingüísticos de los Pueblos Indígenas.
Se puede considerar que la política no tiene injerencia en la formación de la identidad étnica, ya que como dicen algunos autores, en las comunidades indígenas ninguna actividad cotidiana es considera política; sin embargo ellos tienen sus propias y muy características instituciones políticas; la propia Constitución Mexicana en su artículo $2^{\circ}$ señala que los pueblos indígenas deben conservan sus propias instituciones sociales, económicas, culturales y políticas. Según Antonio Higuera, que recoge el concepto del antropólogo social Jorge Alonso Sánchez en las prácticas y en reglamentaciones, así como en las instituciones, la política se va construyendo y estableciendo como parte de la cultura a la cual pertenece" (Ballesteros Pérez, (2006). Los pueblos indígenas tienen sus propias instituciones como el Delegado, el Comisario Ejidal y el Presidente de Bienes Comunales, de igual manera, cuenta con un consejo conformado por los varones de mayor edad de la población dentro del cual las mujeres no pueden participar. Esto lo manifiesta Max Weber en la obra "Gente de Costumbre y Gente de Razón" que menciona "la observación de que la acción 
política compartida construye la comunidad e incluso puede generar la idea de formar parte de una "comunidad de sangre". La vida política influye entonces en la misma definición organizacional del grupo étnico y contribuye a su identidad distintiva" (Bartolomé, (2006).

\section{RELIGIÓN $\quad$ Y RITUALES INDÍGENAS}

"La religión no es más que el reflejo en la mente de los hombres de las fuerzas externas que controlan su vida diaria". (Engels). El mundo religioso no es sino el reflejo del mundo real. $\mathrm{Y}$ el cristianismo, con su culto del hombre abstracto, es el complemento religioso más conveniente. La difusión del catolicismo entre los indígenas, se considera generalmente como la dimensión simbólica de la dominación colonial española (la llamada conquista espiritual) (Beaucage, (2004).

La evangelización fue unos de los procesos de aculturación más importante para la conquista. Sin embargo no se pudo acabar completamente con la cultura de ritos indígenas, así que se entremezclaron los elementos propios de los pueblos originarios con los de la religión católica, y hasta nuestros días persisten. A esta combinación de creencias se les denomina sincretismo, el cual tiene su mejor ejemplo en el pueblo de San Juan Chamula, Chiapas, donde se puede apreciar con sus características muy peculiares, ya que las creencias y la religiosidad conforman factores que determinan la identidad.

El término religión nos viene del latín religió, "escrúpulo, delicadeza”. Para algunas se deriva del verbo relegere, "recoger, repasar, releer"; para otros, en cambio, de la palabra religare, "religar, unir, ceñir", es decir es "reflexión o relectura", como "liga o unión". Esta unión de lo humano y divino proporciona seguridad, certidumbre a las personas que comparten una ideología de vida, además de compartir normas de conducta que les proporciona una misma identidad. Por otro lado Emilio Durkheim considera a lo religioso como aquello que está involucrado con lo sagrado: "una religión es un sistema solidario de creencias y prácticas relativas a cosas sagradas, es decir, separadas, prohibidas; creencias y prácticas que unen en una misma comunidad moral, llamada Iglesia, a todos los que se adhiere a ella". En la 
religiosidad lleva implícito lo sagrado. El término "sagrado" es lo "Digno de veneración por su carácter divino o por estar relacionado con la divinidad", es decir que son cuestiones sobrenaturales de carácter apartado o desconocido (Beaucage, (2004). En opinión de Ricardo e Isabel de Pozas las ideas que conserva el indio relativas a la explicación que se daba en el pasado prehispánicos a la creación y origen del cosmos y del hombre son hoy por hoy un remanente de su religión, pues a veces la simple explicación de un rito pone al descubierto una serie de ideas en las que aparecen entremezclados el hombre, la divinidad, los espíritus y ciertos animales fantásticos (Pozas y De Pozas, (2006).

Entre los ritos se pueden encontrar el uso de alucinógenos con efectos variados según la cultura que lo aplique, como el consumo de hongos en la selva lacandona en Chiapas y en Oaxaca, y el peyote utilizado por los huicholes para entrar en contacto con seres espirituales. De igual manera existen una serie de prácticas entremezcladas en sus fiestas religiosas y patronales ya que rinden culto a divinidades para recibir algún beneficio como lluvias para obtener buenas cosechas o solicitar la salud de algún ser querido; también dentro de las festividades se llevan a cabo la celebración a sus santos patrones y a cualquier otra figura religiosa. Esas prácticas o conductas que se manifiestan por costumbres son los rituales que es un conjunto de ritos de una religión, de una iglesia o de una función sagrada; mientras que los ritos son las reglas establecidas para el culto y ceremonias religiosas. Sin querer entrar a un análisis histórico, la religión aparece como un conjunto de ritos y creencias a los cuales no está ligado ningún sistema moral; los ritos mismos, los sacrificios humanos, la embriaguez y la antropofagia ritualista, eran contrarios a la moral humana; por ejemplo, los aztecas practicaban la comunión con los dioses bajo diversos aspectos, una de sus formas, la absorción del corazón de la víctima, asimila a la sustancia de Dios, bajo la misma idea pero con un aspecto menos sangriento y otra forma era la de comer imágenes hechas de pasta de alimentos que representaban al dios Huitzilopochtli dos veces al año (Ricard, (2002).

El ritual tuvo entre los pueblos indígenas prehispánicos un papel importante como parte de su identidad. 


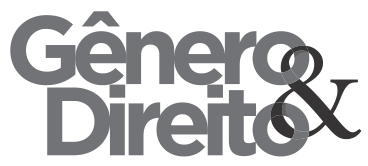

Como podemos apreciar hay una diversidad de rituales que se conservan al día de hoy en las misas católicas; coexisten con la práctica de rituales agrarios de propiciación de lluvias, oferta de primicias y prácticas para la caza. De igual manera existen rituales clandestinos que tienen lugar en cavernas y otros lugares ocultos, cuyo objetivo pueden incluir la petición de la muerte de los adversarios (Bartolomé, (2006).

A pesar de las represiones de las religiones indígenas por parte de los españoles y posteriormente por los criollos y mestizos en busca de homogenizar las creencias e ideología religiosa para formar parte de una misma comunidad, los rituales continúan en el sincretismo de los pueblos indígenas. $\mathrm{Y}$ en palabras de Bartolomé "la participación plena en la comunidad ritual es entonces posible sólo para aquellos que a través de ella ejercen y reactualizan tanto su historia cultural específica como los nexos organizacionales e identitarios que los configuran como grupo étnico. Sin embargo, lo que realmente importa para la pertenencia social no es tanto el conocimiento, como la práctica de la cosmología: el compartir una "costumbre" dico do Núcleo de Estudos e Pesquisas sobre Gênero e Direito Centro de Ciências Jurídicas - Universidade Federal da Paraíba V. 6 - No 03 - Ano 2017 (Spanish Edition)

101

ritual que identifica a los protagonistas más que el conocimiento de su sentido", es decir, es más de comportamiento conductual que de filosofía de Dios y sus virtudes (Bartolomé, (2006).

Los rituales en las sociedades indígenas han sido parte de la temática explorada por la antropología desde los inicios de la construcción como ciencia social, y como tal han tenido exponentes teóricos significativos dentro de las corrientes. En la actualidad, muchos de los estudiosos aceptan que todo ritual, sea religioso o civil, es un sistema de símbolos; una trama de significados o conceptos enunciados en forma codificada y sintética, y rodeados de una fuerte afectividad (Gámez Espinoza, (2015).

\section{CONCLUSIÓN.}

La etnia es la forma de organización de grupos sociales, cuyos mecanismos de delimitación frente a otros grupos con los que mantienen algún tipo de interacción, son definidos por sus miembros a partir de rasgos en común de cada grupo y que se pueden presentar con conductas aculturizadas. 


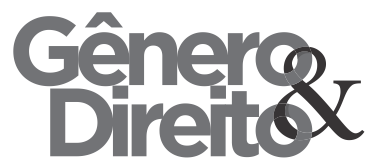

En palabras del autor Miguel

Alberto Bartolomé "la identidad étnica no refiere necesariamente a un momento histórico especifico sino al estado contemporáneo de una tradición, aunque puede desarrollar una imagen ideologizada de sí misma y de su pasado" (Bartolomé, (2006).

Para los filósofos, la cultura se percibe como las manifestaciones superiores de la vida social; mientras que los antropólogos manejan el término cultura con un contenido más amplio, global, que comprende todas las manifestaciones de la vida social, la técnica, los hábitos, creencias, tradiciones y toda la obra material y psíquica del hombre (Pozas y De Pozas, (2006).

Sin embargo, en la actualidad también es necesario para los indígenas que se reconozcan como tal, aunque no cuenten con algunos de los factores que determinan la identidad, pero con el simple hecho de aceptarse como son, miembros de una comunidad, son considerados indígenas. De ahí el reforzamiento de políticas y leyes e instrumentos jurídicos internacionales que los protejan, avalen y propicien el respeto por su cultura y su desarrollo.

\section{REFERENCIAS BIBLIOGRÁFICAS}

Aguilar Rivero, (2012). Hacia una política de las identificaciones, Di Castro, Elisabetta (coord.), et al., Construcción de identidades. México.

Andrea Maidana, (2009). Volver a la tierra, parentesco, redimensionalización territorial y reconstrucción identitaria, Tamagno, Liliana (Coord.). Pueblos indígenas: interculturalidad, colonialidad, política. Buenos Aires, Argentina, Editorial Biblos.

Ballesteros Pérez, (2006). Apuntes etnográficos del estudio de la cultura política, Higuera Bonfil, Antonio (Coord.). Trabajo de campo, la antropología en acción. México. Ed. Plaza y Valdés.

Bartolomé, (2006). Gente de costumbre y gente de razón. Tercera edición. México, Ed. Siglo XXI.

Batzin, C. (2005). El desarrollo humano y los pueblos indígenas. Seminario Internacional Pueblos Indígenas y 


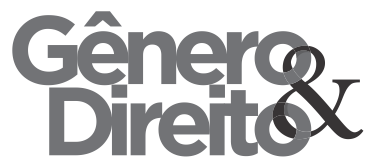

afrodescendientes de América Latina y el

Caribe: relevancia y pertinencia de la Información sociodemográfica para políticas y programas. CEPAL, Santiago de Chile. Recuperado en http://www.cepal.org/celade/noticias/pagi nas/7/21237/CBatzin.pdf

Bauman, (2013). La cultura en el mundo de la modernidad líquida. México. Ed. Fondo de Cultura Económica.

Beaucage, (2004). Pensamiento crítico y nuevos movimientos religiosos indígenas, Castro Lucic, Milka (Coord.). Los desafíos de la interculturalidad: identidad, política y derecho. Santiago de Chile. LOM Ediciones.

Bello, (2004). Etnicidad y ciudadanía en América Latina. La acción colectiva de los pueblos indígenas. Comisión Económica para América Latina y el Caribe (CEPAL). Sociedad Alemana de Cooperación Técnica (GTZ). Santiago de Chile. Recuperado en http://www.cepal.org/mujer/noticias/notic ias/9/26089/libroetnicidadciudadania.pdf
Bertely Busquets, et al., (2013). Adolescentes indígenas en México: derechos e identidades emergentes. México. CIESAS. Fondo de las Naciones Unidas de la Infancia. Recuperado en http://www.unicef.org/mexico/spanish/Ad olescentesindigenasDoctecnicoOK2.pdf

Castro Lucic, (2004). La cuestión intercultural: de la exclusión a la regulación. Los desafíos de la interculturalidad: identidad, política y derecho. Santiago de Chile. LOM Ediciones.

Díaz-Polanco, (2009). Para entender la diversidad cultural y la autonomía en México. México. Nostras ediciones.

Diccionario de la Lengua Española (DRAE), Real Academia Española, $23^{\circ}$ ed. Recuperado en http://lema.rae.es/drae/?val=religi\%C3\%

B3n

Durand Alcántara, (2005). Derecho indígena. Segunda edición. México. Ed. Porrúa. 


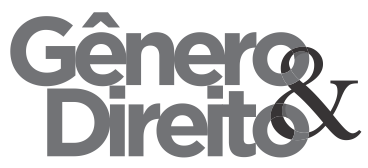

Periódico do Núcleo de Estudos e Pesquisas sobre Gênero e Direito Centro de Ciências Jurídicas - Universidade Federal da Paraíba V. 6 - No 03 - Ano 2017 (Spanish Edition) ISSN | 2179-7137 | http://periodicos.ufpb.br/ojs2/index.php/ged/index
Durand Alcántara, (2006). Derechos de indios en México. Derechos pendientes. México, Ed. Porrúa.

Gámez Espinoza, (2015). Cosmovisión mesoamericana: reflexiones, polémicas y etnográficas. México. Fondo de Cultura Económica.

García Canclini, (2005). Diferentes, desiguales y desconectados. Segunda edición. Barcelona, España. Editorial Gedisa.

Grebe Vicuña, (2010). Culturas indígenas de Chile. Un estudio preliminar. Quinta edición. Chile. Editorial Pehuén.

Instituto de Investigaciones Jurídicas, Enciclopedia Jurídica Mexicana, México, Ed. Porrúa, Tomo V.

Martínez, (s. f.). ¿Cuál es el objetivo real de la política? Cultura General. Lifeder.com. Recuperado en https://www.lifeder.com/objetivopolitica/

Naciones Unidas de Derechos Humanos (1965). Convención Internacional sobre la eliminación de todas las formas de

Discriminación Racial. Recuperado en http://www.ohchr.org/SP/ProfessionalInte $\underline{\text { rest/Pages/CERD.aspx }}$

Naciones Unidas (1995). Acuerdo sobre identidad y derechos de los pueblos indígenas. Guatemala. Recuperado en http://www.guatemalaun.org/bin/docume nts/Acuerdo\%20Pueblos \%20Ind\%C3\%A

Dgenas.pdf

Organización Internacional del Trabajo (OIT), (1989). Convenio sobre Pueblos Indígenas y tribales, (Convenio 169 de la Organización Internacional del Trabajo). Instituto nacional de Lenguas Indígenas (INALI). Recuperado en http://www.inali.gob.mx/pdf/Convenio_1 69_OIT.pdf

Pérez Castro, (2012). Tiempo de emigrar; identidades en construcción. Di Castro, Elisabetta (coord.), et al. Construcción de identidades. México.

Pérez de los Reyes, (2013). Los derechos fundamentales de los indígenas. México. Ed. Porrúa. 
Pérez Monroy, (2012). La moda: signo de identidad. Di Castro, Elisabetta, et al. Construcción de identidades. México.

Pozas, Ricardo y De Pozas, (2006). Los indios en las clases sociales de México. Vigésima primera edición. México. Ed. Siglo XXI.

Ricard, (2002). La conquista espiritual de México. Séptima edición. México. Fondo de Cultura Económica. 